\title{
Post-Synthesis Optimization of Reversible Circuits
}

\author{
Edinelço Dalcumune $^{1,3}$, Luis A. B. Kowada ${ }^{2}$, Celina M. H. de Figueiredo ${ }^{3}$, \\ Franklin de L. Marquezino ${ }^{3}$ \\ ${ }^{1}$ DCEX - Universidade Federal dos Vales do Jequitinhonha e Mucuri (UFVJM) \\ Teófilo Otoni, MG - Brazil \\ ${ }^{2}$ Instituto de Computação - Universidade Federal Fluminense (UFF) \\ Niterói, RJ - Brazil \\ ${ }^{3}$ PESC/COPPE - Universidade Federal do Rio de Janeiro (UFRJ) \\ Rio de Janeiro, RJ - Brazil
}

edcomune@ufvjm.edu.br, luis@ic.uff.br, \{celina, franklin\}@cos.ufrj.br

\begin{abstract}
One of the main motivations for reversible computing is that quantum computing has as one of its foundations the reversibility of all gates, that is, quantum computing circuit models are reversible. An important problem in reversible computing that has been intensively studied for the last decades is the synthesis of reversible circuits. The extended abstract considers optimization rules aiming to a new algorithm for post-synthesis optimization of reversible circuits composed of generalized Toffoli gates.
\end{abstract}

\section{Introduction}

The synthesis of reversible circuits is an important problem in reversible computing and has received considerable attention due to the possibility of applications in many areas of science such as cryptography, quantum computing, low-power design, and bioinformatics $[5,8,11]$. The synthesis depends on the number of gate types (gate library) used in realizing the circuits, and the reversible circuit is defined as a sequence of reversible gates.

Post-synthesis optimization techniques for reversible circuits using Toffoli gates with positive controls (also known as Multiple Control Toffoli or MCT gates) have been used in several studies [7, 8, 13]. Generalized Toffoli gates (also known as MCT gates with mixed controls) were introduced for the synthesis of reversible circuits by [6], and in [3] they were used for the first time for post-synthesis optimization of reversible circuits. In recent years, there has been a growing interest in post-synthesis optimization of reversible circuits using Toffoli gates with negative controls via substitution rules and template matching $[1,2,3,4,9,10,12,14,15]$.

In this extended abstract, we shall focus on reviewing the rules of $[1,2,4,10,14]$, in order to propose a new algorithm for post-synthesis optimization of reversible circuits using generalized Toffoli gates. The number of gates or Gate Count (GC) is the metric used to evaluate our post-synthesis optimization process.

\section{Optimization rules}

Simplification rules are given to optimize reversible circuits using a post-synthesis algorithm. We give two elimination, as well as merge, swap, move and replacement rules. 
Rule 1 (Elimination of Identical gates) Two identical generalized Toffoli gates $g$ and $h$ cancel each other $[1,2,8,10]$.

Rule 2 (Elimination of NOT gates) Assume that two NOT gates on a line $y$, with no target placed on line $y$ in any of the gates in between, are given. We can remove the NOT gates and complement the polarities of all control connection on line y between the two NOT gates [1, 3, 4].

Definition 1 Two generalized Toffoli gates $g$ and h are adjacent if they have the targets on the same line, and differ in only one line with respect to the controls (positive, negative or "don't care").

Rule 3 (Merge for Adjacent gates) Two adjacent gates can be merged into one gate in the reversible circuit. The resulting gate will have the control connection (positive, negative or "don't care") which does not appear on the line in which they differ [1, 2, 3, 4, 10].

Rule 4 (Swap) Two generalized Toffoli gates can be swapped if one of the following conditions occurs: (i) the target line of one gate is not a control line of the other gate; (ii) the two gates have a reverse control connection on one line, and in all other lines on which both gates have control connections, these connections are identical [1, 2, 10, 14].

Rule 5 (Move) Let $g$ and $h$ be two generalized Toffoli gates and suppose the following conditions are satisfied: (i) the target of gate $g$ is on a line where gate h has a control connection, and (ii) every line on gate $g$ with a control connection also has a control connection on gate $h$, and in all lines on which both have control connections these are the same. Then $g$ and $h$ can be switched if we invert the control connection of the $h$ gate positioned on the same line as the target of the g gate [2].

Definition 2 Two generalized Toffoli gates $g$ and h are distance-2 gates if they have the targets on the same line, and differ in two lines with respect to the controls (positive, negative or "don't care"). We refer to Table 1 where we depict the possible scenarios.

Rule 6 (Replacement for Distance-2 gates) A pair of distance-2 gates $g$ and $h$ can be replaced by other pairs of distance-2 gates according to Table 1, which shows the possibilities for replacement, where the previously known cases [4] are referenced. In each set of three pairs of gates, we can choose the most appropriate pair of gates.

Table 1 depicts the six possible different sets with three equivalent pairs of gates. In each set of gates we can choose the most appropriate pair of gates for the reversible circuit, i.e., a pair of gates that leads to a new application of rules, possibly providing a decrease in the gate count.

Lemma 1 There are eighteen types of pairs of distance-2 gates, divided into six sets of three elements each. The three pairs of gates in each set are equivalent.

\section{Proposed algorithm}

To describe Algorithm 1 that explores for the first time the six above described rules simultaneously, we need the concept of segment. In a given reversible circuit of generalized Toffoli gates $G=\left\{g_{1}, g_{2}, \ldots, g_{p}\right\}$, a segment is defined as a set of consecutive gates $G_{\text {seg }}=\left\{g_{k_{1}}, g_{k_{1}+1}, \ldots, g_{k_{2}}\right\}$, where $G_{\text {seg }} \subseteq G, 1 \leq k_{1} \leq k_{2} \leq p$, such that any pair of gates in $G_{\text {seg }}$ can be swapped without affecting the functionality of the circuit. 
Table 1. Scenarios for distance-2 gates

\begin{tabular}{|c|c|c|c|c|c|c|c|}
\hline \multirow{2}{*}{ Case } & \multirow{2}{*}{ Line } & \multicolumn{2}{|c|}{ Pair 1} & \multicolumn{2}{|c|}{ Pair 2} & \multicolumn{2}{|c|}{ Pair 3} \\
\hline & & $g$ & $h$ & $g$ & $h$ & $g$ & $h$ \\
\hline \multirow{2}{*}{$a[4]$} & $\mathrm{i}$ & 1 & 0 & 1 & - & 0 & - \\
\hline & $\mathrm{j}$ & 0 & 1 & - & 1 & - & 0 \\
\hline \multirow{2}{*}{$b[4]$} & $\mathrm{i}$ & 1 & 0 & 1 & - & 0 & - \\
\hline & $\mathrm{j}$ & 0 & - & 1 & -1 & 1 & 0 \\
\hline \multirow{2}{*}{$c[4]$} & $\mathrm{i}$ & 1 & - & 0 & - & 1 & 0 \\
\hline & $\mathrm{j}$ & 0 & 1 & 0 & -1 & - & 1 \\
\hline \multirow{2}{*}{$d[4]$} & $\mathrm{i}$ & 1 & - & 0 & -1 & 1 & 0 \\
\hline & $\mathrm{j}$ & 1 & 0 & 1 & -1 & - & 0 \\
\hline \multirow{2}{*}{$e$} & $\mathrm{i}$ & 1 & 0 & 1 & -1 & 0 & - \\
\hline & $\mathrm{j}$ & 1 & - & 0 & - & 0 & 1 \\
\hline \multirow{2}{*}{$f$} & $\mathrm{i}$ & 1 & - & 1 & 0 & 0 & - \\
\hline & $\mathrm{j}$ & - & 0 & 1 & 0 & - & 1 \\
\hline
\end{tabular}

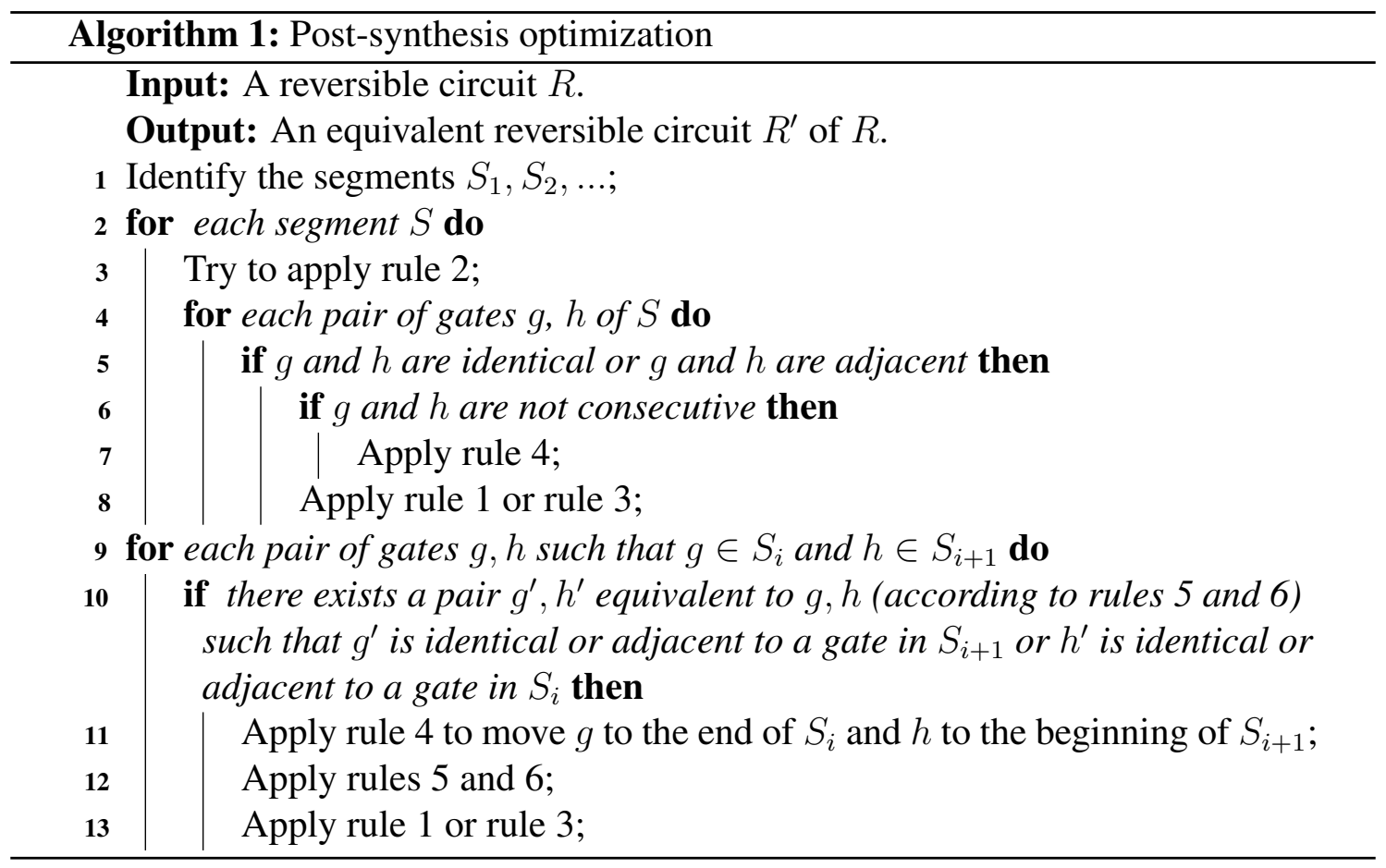




\section{References}

[1] A. A. A. de Almeida, G. W. Dueck, and A. C. R. da Silva. Reversible circuit optimization based on tabu search. In IEEE Int. Symp. on Mult-Valued Log., pages 103-108, 2018.

[2] X. Cheng, Z. Guan, W. Wang, and L. Zhu. A simplification algorithm for reversible logic network of positive/negative control gates. In Int. Conf. on Fuzzy Syst. and Knowledge Discovery, pages 2442-2446, May 2012.

[3] K. Datta, G. Rathi, R. Wille, I. Sengupta, H. Rahaman, and R. Drechsler. Exploiting negative control lines in the optimization of reversible circuits. In Gerhard W. Dueck and D. Michael Miller, editors, Reversible Computation, pages 209-220. Springer, 2013.

[4] K. Datta, I. Sengupta, and H. Rahaman. A post-synthesis optimization technique for reversible circuits exploiting negative control lines. IEEE Trans. Comput., 64(4):1208-1214, 2015.

[5] L. A. B. Kowada, R. Portugal, and C. M. H. de Figueiredo. Reversible Karatsuba's algorithm. J. Univers. Comput. Sci., 12(5):499-511, jun 2006.

[6] D. Maslov and G. W. Dueck. Level compaction in quantum circuits. In IEEE Int. Conf. on Evol. Comput., pages 2405-2409, 2006.

[7] D. Maslov, G. W. Dueck, and D. M. Miller. Toffoli network synthesis with templates. IEEE Trans. Comput.-Aided Design Integr. Circuits Syst., 24(6):807-817, 2005.

[8] D. M. Miller, D. Maslov, and G. W. Dueck. A transformation based algorithm for reversible logic synthesis. In Ann. Design Aut. Conf., pages 318-323. ACM, 2003.

[9] M. M. Rahman, M. Soeken, and G. W. Dueck. Dynamic template matching with mixedpolarity Toffoli gates. In IEEE Int. Symp. on Mult-Valued Log., pages 72-77, 2015.

[10] Md Z. Rahman and J. E. Rice. Templates for positive and negative control Toffoli networks. In Shigeru Yamashita and Shinichi Minato, editors, Reversible Computation, pages 125-136. Springer, 2014.

[11] M. Saeedi and I. L. Markov. Synthesis and optimization of reversible circuits - a survey. ACM Comput. Surv., 45(2):21:1-21:34, mar 2013.

[12] M. Soeken and M. K. Thomsen. White dots do matter: Rewriting reversible logic circuits. In Gerhard W. Dueck and D. Michael Miller, editors, Reversible Computation, pages 196-208. Springer, 2013.

[13] M. Soeken, R. Wille, G. W. Dueck, and R. Drechsler. Window optimization of reversible and quantum circuits. In IEEE Symp. on Design and Diag. of Elect. Circuits and Syst., pages 341-345, 2010.

[14] R. Wille, M. Soeken, C. Otterstedt, and R. Drechsler. Improving the mapping of reversible circuits to quantum circuits using multiple target lines. In Asia and South Pacific Design Aut. Conf., pages 145-150, 2013.

[15] R. Wille, M. Soeken, N. Przigoda, and R. Drechsler. Exact synthesis of Toffoli gate circuits with negative control lines. In IEEE Int. Symp. on Mult-Valued Log., pages 69-74, May 2012. 